\title{
Visual loss secondary to fludarabine toxicity: optical coherence tomography findings in two patients
}

\author{
Jonathan Virgo $^{1} \cdot$ Graeme Williams $^{2} \cdot$ Miles Stanford $^{1}$ \\ Received: 19 December 2018 / Accepted: 7 January 2019 / Published online: 28 January 2019 \\ (c) The Royal College of Ophthalmologists 2019
}

Fludarabine is a purine analogue used to treat haematological malignancy and for conditioning prior to allogenic bone marrow transplant. In early clinical trials [1] high-dose fludarabine caused high rates of severe neurotoxicity $(56 \%)$ and death $(44 \%)$. Fludarabine is now used at lower doses in combination with other chemotherapy drugs (e.g. FLAG-Ida regimen). However, the risk of severe sight loss and blindness in one study [2] was $1 \%$ and $0.3 \%$, respectively.
We present two patients with painless loss of vision secondary to fludarabine toxicity. Both had normal ophthalmic examination and optical coherance tomography (OCT) at presentation. Patient 1 developed blurred vision 14 days after FLAG-Ida cycle 2. At presentation visual acuities (VA) bilaterally were $6 / 18$. Six weeks later VA worsened to $6 / 36$ and high-dose oral prednisolone was prescribed. Initially VA improved to $6 / 9$ bilaterally, but declined to $6 / 60$ over 3 years.

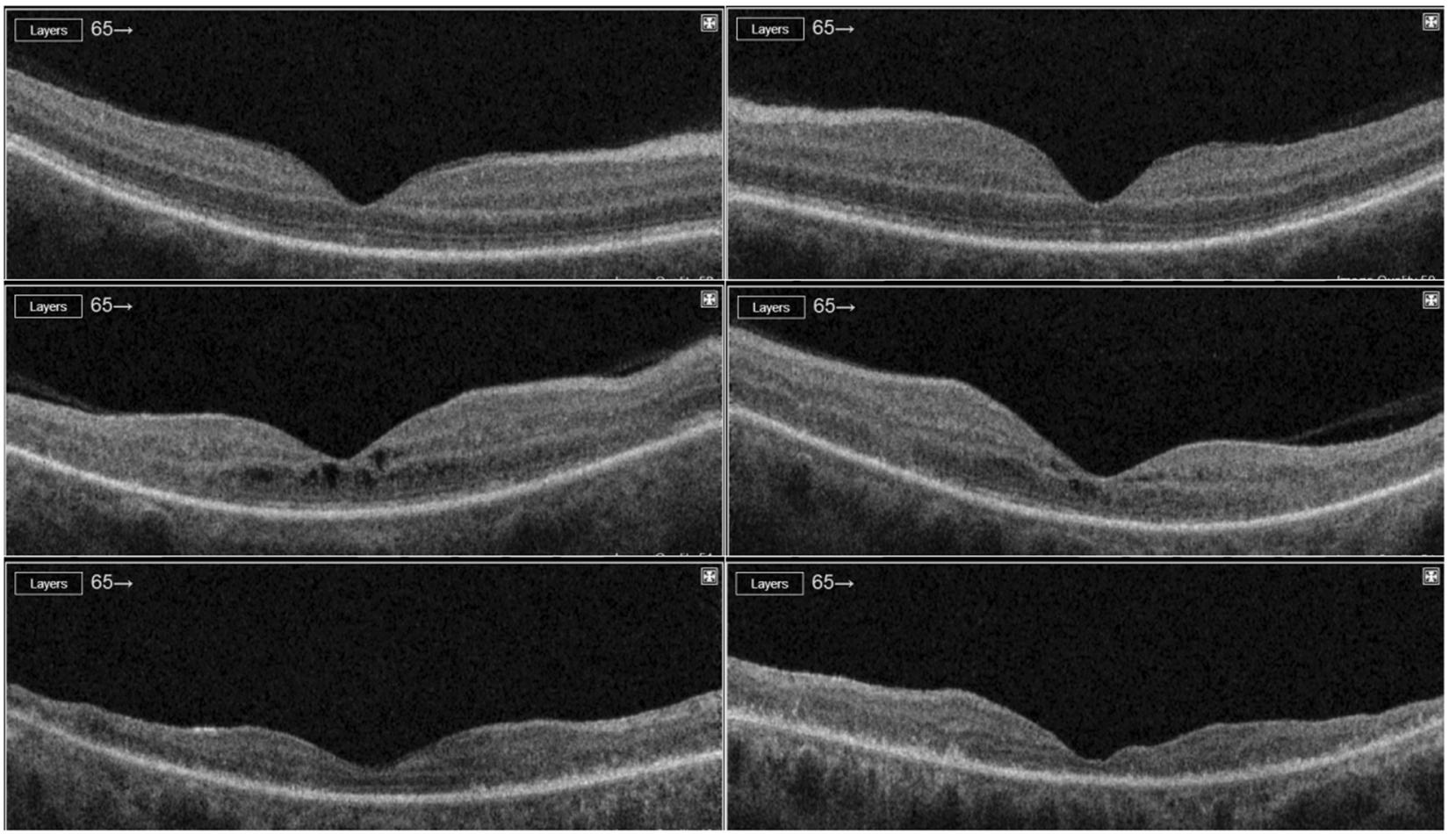

Fig. 1 Macular optical coherence tomography from patient 1 at 30 days post chemotherapy (top), 10 months post chemotherapy (middle) and 3 years post chemotherapy (bottom)

Jonathan Virgo

jdvirgo@nhs.net
Medical Eye Unit, St Thomas' Hospital, London SE1 7EH, UK

2 Ophthalmology Department, Gartnavel General Hospital, Glasgow G12 0YN, UK 
Patient 2 experienced dimming of vision during FLAG-Ida cycle 1, with VA 6/9 bilaterally. Despite a healthy ocular surface, keratoconjunctivitis secondary to cytarabine was diagnosed and the dose halved. During cycle 2 VA dropped to perception of light in both eyes, but recovered in $<1$ week to 6/9 with high-dose oral prednisolone and remained stable throughout 8 months' follow-up.

Punctate yellow intraretinal macular flecks, previously reported with fludarabine toxicity [3], were not observed in our patients. OCT features have not previously been reported. Electronegative electroretinogram (ERG) and bipolar cell dysfunction are previously reported findings $[3,4]$. Histopathology shows loss of retinal ganglion cells, loss of bipolar cells and inner nuclear layer oedema; inner retina and optic nerve head gliosis and inflammatory cell infiltrate are also reported [3, 4]. Fludarabine can also cause brain white matter toxicity [5], affecting the occipital lobes with central visual loss.

Initially, bright flash rod ERGs from patient 1 were electronegative with reduced oscillatory potentials (OPs), indicating inner retina dysfunction. Over time the B wave improved, but OPs did not. Multifocal ERGs were globally reduced and delayed, and worsened over 2 years. OCTs, normal at presentation, over time showed microcystic macular oedema (inner nuclear layer oedema), outer nuclear layer oedema and photoreceptor layer disruption involving the external limiting membrane and ellipsoid zone, along with generalised retinal thinning (Fig. 1).

Bright flash rod ERGs from patient 2 were also electronegative. Pattern ERGs showed a low N95/P50 ratio suggesting retinal ganglion cell dysfunction. OCTs, normal at presentation, developed microcystic macular oedema, outer
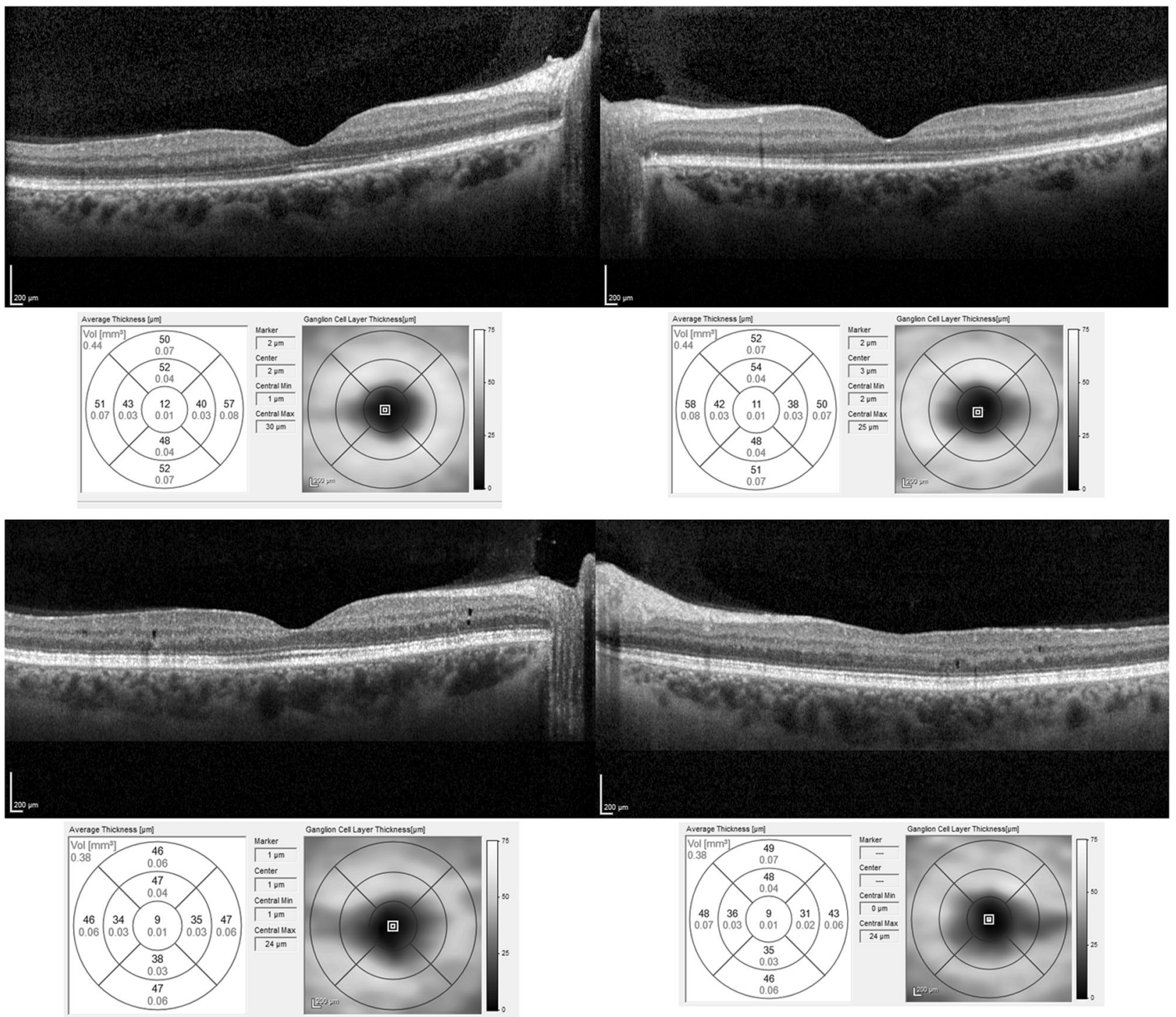

Fig. 2 Macular optical coherence tomography from patient 2 at 4 days post chemotherapy (top two rows) and 8 months post chemotherapy (bottom two rows) 
nuclear layer oedema and thinning of the ganglion cell layer during follow-up (Fig. 2). At presentation magnetic resonance imaging (MRI) showed subtle white matter changes in the occipital lobes.

Fludarabine-related visual loss is well documented and our patients had several classic features. Diagnosis must be made based on history, as initial examination and OCT are normal. Fludarabine must be stopped when toxicity is suspected and investigated with ERG and brain MRI. This report is the first to include OCT. Serial OCTs during follow-up show the evolution of various retinal changes (see above), most of which have previously been shown via histopathology in case reports. There is currently no good quality evidence to guide treatment.

\section{Compliance with ethical standards}

Conflict of interest The authors declare that they have no conflict of interest.
Publisher's note: Springer Nature remains neutral with regard to jurisdictional claims in published maps and institutional affiliations.

\section{References}

1. Warren RP, Jr, Berman E. Phase I and II study of fludarabine phosphate in leukemia: therapeutic efficacy with delayed central nervous system toxicity. J Clin Oncol. 1986;4:74-9.

2. Sorensen, et al. Treatment of refractory chronic lymphocytic leukemia with fludarabine phosphate via the Group C Protocol Mechanism of the National Cancer Institute: five-year follow-up report. J Clin Oncol. 1997;15:458-65.

3. Bishop, et al. Rapid vision loss associated with fludarabine administration. Retina. 2010;30:1272-7.

4. Ding, et al. Ocular toxicity of fludarabine: a purine analog. Expert Rev Ophthalmol. 2008;3:97-109.

5. Lee, et al. Clinical and Imaging Features of Fludarabine Neurotoxicity. J Neuroophthalmol. 2010;30:37-41.

\title{
Needles as a source of silicone oil during intravitreal injection
}

\author{
Gustavo Barreto Melo $\mathbb{1}^{1,2} \cdot$ Geoffrey Guy Emerson ${ }^{3} \cdot$ Acácio Alves Souza Lima Filho ${ }^{2} \cdot$ Shoko Ota ${ }^{4}$ Maurício Maia ${ }^{2}$
}

Received: 20 December 2018 / Accepted: 21 December 2018 / Published online: 13 February 2019

(c) The Royal College of Ophthalmologists 2019

\section{Introduction}

The presence of silicone oil (SO) droplets in the vitreous, even though frequently considered harmless, has been reported in $0.03-1.7 \%$ of eyes receiving intravitreal injections [1].

The source of these droplets is supposedly the syringes, since they are coated with $\mathrm{SO}$ in order to facilitate the

Gustavo Barreto Melo

gustavobmelo@yahoo.com.br

Hospital de Olhos de Sergipe, Aracaju, SE, Brazil

Federal University of São Paulo, São Paulo, SP, Brazil

Retina Center of Minnesota, Minneapolis, MN, USA

4 Chemical Analysis Laboratory, Center for Chemistry and Manufactured Goods, Institute for Technological Research, São Paulo, Brazil

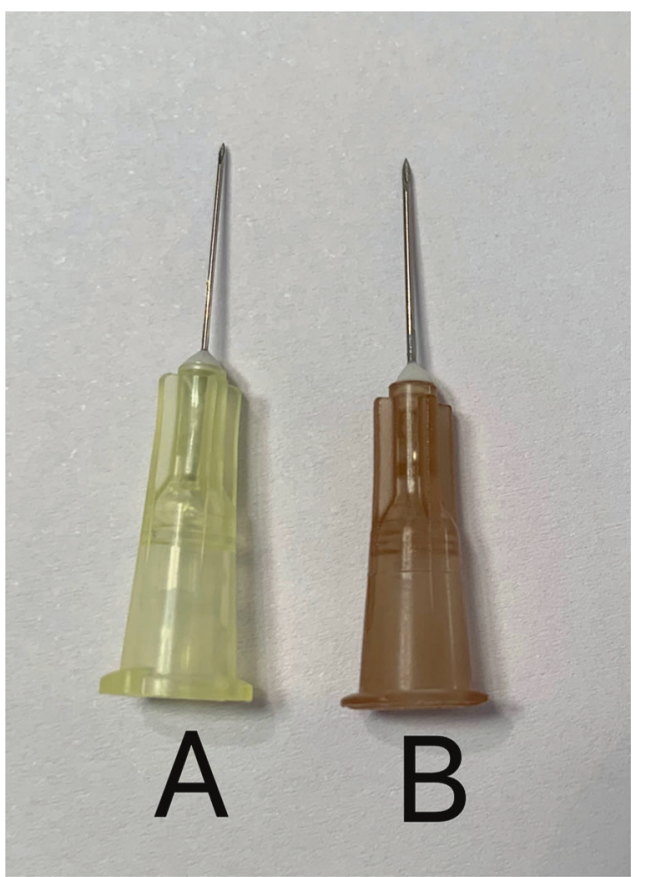

Fig. 1 BD PrecisionGlide needles. a 30-gauge needle; b 26-gauge needle 